\title{
Hipotermia Acidental: Implicações para os cuidados de enfermagem no transoperatório
}

\author{
Accidental Hypothermia: Implications for nursing care during surgery \\ Hipotermia Accidental: Implicaciones para los cuidados de enfermería en el \\ transoperatorio
}

Gisele Santana Muniz ${ }^{1}$, Naracelia Sousa Barbosa Teles ${ }^{2}$, Ilse Maria Tigre de Arruda Leitão ${ }^{3}$, Paulo Cesar de Almeida ${ }^{4}$, Marcelo Chagas Leitão ${ }^{5}$

\begin{abstract}
RESUMO: Objetivo: identificar as intervenções de enfermagem no transoperatório para prevenção e/ou tratamento da hipotermia acidental. Método: pesquisa exploratória, descritiva com abordagem quantitativa, realizada em um centro cirúrgico de hospital público da rede estadual de Fortaleza-CE. A amostra constituiu-se de 12 intervenções realizadas pela equipe de enfermagem em pacientes submetidos a 120 procedimentos cirúrgicos, de janeiro a junho de 2011. Resultados: constatou-se que a temperatura média na sala de admissão, do forno para aquecimento, do recipiente para armazenamento de fluidos aquecidos e da sala de cirurgia não estavam em concordância com aquelas recomendadas. Conclusão: os resultados demonstraram a necessidade de intervenções eficazes para a prevenção da hipotermia e que o Enfermeiro tem papel importante neste contexto, uma vez que a segurança do paciente e a redução de complicações decorrentes do procedimento anestésico-cirúrgico são metas do cuidado de enfermagem.
\end{abstract}

PALAVRAS-CHAVE: Enfermagem. Hipotermia. Perioperatório.

ABSTRACT: Objective: to describe the nursing interventions in surgical anesthetic procedures in the intraoperative period for the prevention and/or treatment of accidental hypothermia. Method: a descriptive research with quantitative methodological approach carried out in a surgery center of a public hospital in Fortaleza, Ceara state. The study sample comprises 12 nursing interventions conducted by the nurses, auxiliaries, or technicians who assisted patients undergoing surgery in 120 surgical procedures, between January and March 2011. Results: it was possible to verify that the temperatures of the admission room, heating oven, storage container for heated fluids, and operating room were not in compliance with the recommended standards. Conclusion: results showed that there is a need to implement effective interventions to prevent hypothermia and that the nursing staff plays an important role in this context, because patient safety and decreased complications owing to surgical anesthetic procedures are nursing care goals.

KEYWORDS: Hypothermia. Nursery. Perioperative.

RESUMEN: Objetivo: identificar las intervenciones de enfermería en el transoperatorio para prevención y/o tratamiento de la hipotermia accidental. Método: estudio exploratorio, descriptivo con enfoque cuantitativo, realizado en un centro quirúrgico de un hospital público de la red estatal de Fortaleza-CE. Método: la muestra se formó a partir de 12 intervenciones de enfermería aplicadas por el equipo de enfermería que atendió a pacientes sometidos a cirugías en 120 procedimientos quirúrgicos, realizadas de enero a junio de 2011 . Resultados: se constató que la temperatura media en la sala de admisión, la temperatura del horno para calentamiento, la del recipiente para almacenamiento de fluidos calentados y la del quirófano no estaban conforme a las recomendadas. Conclusión: los resultados mostraron la necesidad de intervenciones eficaces para la prevención de hipotermia y, también, el importante papel del enfermero en ese contexto, ya que la seguridad del paciente y la reducción de complicaciones derivadas del procedimiento anestésico-quirúrgico son el objetivo de los cuidados de enfermería.

PALABRAS CLAVE: Enfermería. Hipotermia. Perioperatorio.

\footnotetext{
${ }^{1}$ Enfermeira. Pós-Graduada em Enfermagem em Terapia Intensiva. Universidade Estadual do Ceará. Rua Padre Guerra, 2275 apto. 201. Bairro Parquelândia. CEP 60455-360. Fortaleza, CE, Brasil.

E-mail: gisele.muniz@hotmail.com

${ }^{2}$ Professora. Curso de Graduação em Enfermagem. Faculdade Metropolitana de Fortaleza. Mestre em Farmacologia. Faculdade Federal do Ceará. Av. Conselheiro Gomes de Freitas, 3869. CEP 60833-104. Fortaleza, CE, Brasil.

E-mail: naraceliasbteles@hotmail.com

${ }^{3}$ Professora Assistente. Universidade Estadual do Ceará. Mestre em Políticas de Saúde. Universidade Estadual do Ceará. Av. Engenheiro Santana Junior, 2947 apto. 301. CEP 60192-205. Fortaleza, CE, Brasil.

Telefone: (85) 9989-9931.

E-mail: ilsetigretigre@hotmail.com

${ }^{4}$ Professor Adjunto. Universidade Estadual do Ceará. Doutor em Saúde Pública. Faculdade de Saúde Pública da USP. Rua Carlos Vasconcelos, 636 apto. 1002. CEP 60115-170. Fortaleza, CE, Brasil. E-mail: pc49almeida@gmail.com

${ }_{5}^{5}$ Médico Anestesiologista Especialista em Medicina do Trabalho. Av. Engenheiro Santana Junior, 2947 apto. 301. CEP 60192-205. Fortaleza, CE, Brasil.

E-mail: marcelocleitao@hotmail.com
} 


\section{Introdução}

A hipotermia perioperatória é uma complicação frequente em qualquer tipo de cirurgia e pode ser induzida e acidental ou inadvertida. A hipotermia acidental ou inadvertida é um evento comum em procedimentos anestésico-cirúrgicos, principalmente no pós-operatório, sendo causada pela inibição do centro termorregulador do hipotálamo, o que leva a uma temperatura corporal central menor do que $36^{\circ} \mathrm{C}^{1}$.

A temperatura corporal central é um dos parâmetros fisiológicos mais rigorosamente controlados do organismo. O controle da temperatura corporal dá-se pelo equilíbrio entre a produção e a perda de calor. O hipotálamo é o principal centro regulador da temperatura corporal, integrando os impulsos térmicos provenientes da superfície cutânea e dos tecidos profundos, assegurando um equilíbrio entre a perda e a produção de calor ${ }^{2}$. Na prática de enfermagem, frequentemente se afere a temperatura corporal como parte da rotina hospitalar, já que é um dos sinais vitais do paciente, juntamente com pulso, respiração e pressão arterial. A avaliação da temperatura permite uma estimativa, a mais exata possível, do estado térmico interno de uma pessoa.

A hipotermia é definida como temperatura corporal central menor do que $36^{\circ} \mathrm{C}^{3,4}$; porém, alguns já consideram hipotermia quando a temperatura corporal cai abaixo de $35^{\circ} \mathrm{C}^{5}$. O organismo humano tem sua temperatura central (coração, pulmão, cérebro e órgãos esplâncnicos) entre $36,6^{\circ} \mathrm{C}$ e $37,6^{\circ} \mathrm{C}$, e sua manutenção é primordial para a homeostase.

A hipotermia acidental ou primária é definida como decorrente da redução espontânea da temperatura central. Pode estar associada com disfunções orgânicas agudas ou doenças crônicas agudizadas. Ela advém de vários fatores isolados ou associados, como perda excessiva de calor, inibição da termorregulação fisiológica ou falta de cuidados adequados para sua prevenção. As causas mais frequentes são exposição ao frio e ao vento; infusão excessiva de líquidos parenterais frios, em especial transfusões sanguíneas; imersão em ambientes gelados e umidade excessiva, entre outras ${ }^{3}$. A hipotermia secundária se caracteriza por disfunções ou lesões do centro termorregulador, causadas por doenças orgânicas ou uso de substâncias com ação no sistema nervoso central, incluindo aqui os anestésicos.

O tipo de anestesia e dos fármacos, bem como a natureza cirúrgica repercutem tanto no gradiente de temperatura como no tempo que o paciente ficará na Sala de Recuperação PósAnestésica (SRPA), sendo a indução anestésica a responsável pela redução de $20 \%$ na produção metabólica de calor, além de abolir as respostas fisiológicas termorreguladoras normalmente desencadeadas pela hipotermia ${ }^{6}$. Cirurgias com maior exposição de cavidades e órgãos centrais também podem gerar maior perda de calor. Durante o procedimento anestésico-cirúrgico, além de ocorrerem as alterações induzidas pelos fármacos sobre a fisiologia da termorregulação, a exposição do corpo ao ambiente cirúrgico gera perda de calor para o ambiente por quatro mecanismos: irradiação, condução, evaporação e convecção.

A hipotermia terapêutica, também chamada de 'provocada', é instituída, conscientemente, pela equipe médica, com objetivos bem definidos: tratamento de hipertensão intracraniana refratária, proteção neurológica pós-ressuscitação cardiopulmonar e durante cirurgias neurológicas ou cardíacas de maior complexidade, bem como em algumas afecções que cursam com elevação descontrolada de temperatura, dentre outros.

A hipotermia é um evento comum no perioperatório, ocorrendo em mais de $70 \%$ dos pacientes submetidos ao procedimento anestésico-cirúrgico; pode acarretar complicações relevantes ${ }^{7}$, sendo que as principais descritas na literatura são: aumento da taxa de morbidade; aumento da incidência de infecção do sítio cirúrgico; aumento na demanda cardíaca e de oxigenação na presença de tremores, e prejuízos da função plaquetária.

A prevenção da hipotermia é muito importante, pois visa a impedir que seus efeitos negativos prejudiquem a recuperação dos pacientes. Para evitar o desenvolvimento de hipotermia inadvertida no intra e no pós-operatório, podem ser utilizados métodos que limitem a perda de calor cutâneo para o ambiente ${ }^{4}$. Os métodos utilizados para prevenir o desenvolvimento de hipotermia podem ser divididos em ativo de aquecimento cutâneo ou passivo. Os métodos considerados sistema ativo de aquecimento cutâneo incluem: colchões térmicos com circulação de água, infusão de soluções aquecidas, aquecimento e a umidificação dos gases administrados. $\mathrm{O}$ aquecimento passivo consiste em cobrir e aquecer, durante o intraoperatório, toda superfície cutânea possível com o emprego de lençóis, cobertores ou mantas, o que reduz a perda de calor em $36 \%$.

A literatura diverge em relação ao método mais eficaz para o aquecimento do paciente. Os considerados mais efetivos são aqueles que empregam fornecimento ativo de calor e transferência de energia térmica, principalmente através da manta de ar aquecido, que mantém a temperatura corporal próxima ou igual à normotermia9 .

Aproximadamente $90 \%$ da perda de calor do organismo, durante $o$ ato cirúrgico, ocorre pela superfície da pele, através de irradiação e convecção, associada à evaporação no sítio cirúrgico. Para que seja efetivo, qualquer dispositivo, que previna ou trate a hipotermia, se faz importante a intervenção do profissional Enfermeiro em sala de cirurgia.

Em vários estudos, há a necessidade de avaliação do Enfermeiro em relação à temperatura do paciente e sua monitoração durante todo o transcorrer do procedimento anestésico-cirúrgico. Recomenda-se a implementação de medidas de aquecimento como o uso de cobertor, meias, capuz, exposição limitada da pele, utilização de aparelho de aquecimento artificial, administração de soluções intravenosa e de irrigação aquecidas, umidificação e aquecimento dos gases anestésicos ${ }^{1-3 .}$. 
Diante do exposto acerca da hipotermia acidental ocorrida em pacientes submetidos a cirurgias e do processo de enfermagem empregado como metodologia assistencial, surge um questionamento: quais as intervenções de enfermagem aplicadas para prevenir e controlar a hipotermia?

Buscando responder a esse questionamento, o objetivo deste estudo foi identificar as intervenções de enfermagem em procedimentos anestésicos cirúrgicos no transoperatório para prevenção e/ou tratamento da hipotermia acidental.

Frente à responsabilidade do Enfermeiro em monitorar, controlar e avaliar a temperatura do paciente em sala de cirurgia para a prevenção da hipotermia acidental e de seus efeitos deletérios, torna-se relevante proceder a este estudo, contribuindo, assim, para a promoção de uma assistência segura e de qualidade ao paciente no perioperatório.

\section{Método}

Trata-se de estudo descritivo,exploratório com abordagemquantitativa, que se relaciona a descrição, análise e interpretação dos fenômenos das atividades de enfermagem em sala de cirurgia, relativas a controle, monitoramento e avaliação da temperatura do paciente cirúrgico. $\mathrm{O}$ estudo foi realizado no período de janeiro a junho de 2011, no Centro Cirúrgico de um hospital público de referência da rede Estadual no município de Fortaleza, em nível terciário. O Hospital tem atendimento geral, tanto cirúrgico quanto clínico, abrangendo cirurgias eletivas e de emergências. Possui 16 salas operatórias para realização, em média, de 25 procedimentos por dia. As salas operatórias possuem características -, dimensão, controle de temperatura, acabamento, presença de sistema de ar condicionado - dentro dos padrões recomendados pela $\mathrm{RDC} n$. $^{\circ} 50$. Em duas salas, há dispositivo externo para aquecimento.

A amostra foi constituída por 12 intervenções de enfermagem, que são: controle do uso de líquido para a degermação da pele; controle do uso de fluidos intravenosos e de irrigação cavitária; controle da temperatura de sala; exposição da superfície corporal, método aplicado para aquecer o paciente; temperatura de armazenamento de fluidos; controle da temperatura de forno; controle dos sinais vitais, e controle da diurese. Essas intervenções são realizadas por toda a equipe de enfermagem: Enfermeiros, Auxiliares ou Técnicos, em 120 procedimentos cirúrgicos, que prestaram assistência a pacientes que se submeteram a procedimentos anestésico-cirúrgicos.

O Instrumento utilizado para a coleta de dados foi elaborado sob a forma de um formulário, contendo dados relacionados ao paciente, à prevenção e ao tratamento da hipotermia no transoperatório, fundamentados em um protocolo validado de autoria de Santos e Caregnato ${ }^{1}$ :

- 1. ${ }^{\text {a }}$ fase: Sala de admissão. Foram coletados os dados de identificação, como número de prontuário e sexo, e a presença de morbidades, através dos dados descritos em prontuário. Foram também observadas as intervenções realizadas pela equipe de enfermagem com o intuito de prevenir a hipotermia. Para registrar estes dados, foi utilizado um formulário estruturado. Em seguida, verificava-se a temperatura desta área de recepção com o auxílio de um termômetro de ambiente com sensor externo, próprio da Instituição, termo-higrômetro nacional de marca INCOTERM $\AA$;

- 2. ${ }^{\text {a }}$ fase: Verificação da temperatura do forno de banhomaria. Registrou-se o valor da temperatura que estava programada no painel do forno utilizado pela instituição para aquecer os fluidos, antes de iniciar os procedimentos cirúrgicos; esses fluidos eram colocados no forno sem o controle de temperatura permanente;

- 3. ${ }^{a}$ fase: Observação. Quando o paciente era encaminhado para a sala operatória, continuava-se a observar, durante o procedimento anestésico-cirúrgico, as intervenções da equipe de enfermagem para prevenir a hipotermia;

- 4. ${ }^{a}$ fase: Temperatura do armazenamento de fluidos. Através do termômetro ambiente (termo-higrômetro nacional de marca INCOTERM $\left.{ }^{\circledR}\right)$, o sensor externo era colocado dentro do recipiente de isopor durante 15 a 20 minutos para verificar a temperatura; os fluidos eram armazenados em um recipiente de isopor colocado próximo às salas cirúrgicas.

A análise dos dados foi realizada na forma descritiva. A técnica de dupla digitação foi utilizada e os dados coletados foram processados no SPSS versão 17.0. Para a análise das variáveis quantitativas 'idade' e 'temperatura' foram calculados os valores: máximos, mínimos, médias e desvio padrão. Para a análise de associação de variáveis, foi usado o teste de razão de verossimilhança e o teste do qui-quadrado $\left(\chi^{2}\right)$. Antes dos testes estatísticos, foi verificada a normalidade das variáveis, bem como a igualdade de variâncias. $\mathrm{O}$ nível de significância adotado foi de $5 \%(\mathrm{p}<0,05)$ para todas as análises.

A pesquisa foi conduzida com a aprovação do Comitê de Ética do hospital em estudo, sob o número de protocolo n. ${ }^{\circ}$ 090201/11.

\section{Resultados e Discussão}

A média da idade dos pacientes assistidos pela enfermagem nos 120 procedimentos cirúrgicos foi de 42,3, com desvio padrão de 15,8 anos; não foi possível saber a idade de um paciente, pois o mesmo era desorientado e não foi encontrado tal dado no prontuário. Quanto ao sexo, $72(60 \%)$ pacientes eram do sexo feminino e 48 (40\%) do sexo masculino.

A despeito da clientela atendida pelo serviço, o sexo feminino foi a variável preponderante (72-60\%). Esse é um dado importante para ser identificado como um fator de risco para o desenvolvimento da hipotermia, pois a mulher experimenta menor perda de calor, no período perioperatório, do que o homem, já que seu corpo possui maior percentual de tecido adiposo, que atua como uma camada protetora; por outro lado, a mulher tem menor quantidade de massa 
muscular e maior índice de superfície corporal, podendo ser mais susceptível a perdas de calor para o ambiente.

Dos 120 procedimentos cirúrgicos investigados, quatro foram em pacientes portadores de cardiopatia; dez em portadores de alguma doença neurológica; 29 em hipertensos, e 13 em diabéticos. Em relação às doenças crônicas e ao sexo, dos pacientes hipertensos, foram $18(25 \%)$ do sexo feminino e $11(23 \%)$ do sexo masculino; dos pacientes diabéticos, nove $(12,5 \%)$ eram do sexo feminino e 4 quatro $(8,5 \%)$ do sexo masculino; dos pacientes cardiopatas, dois $(2,8 \%)$ eram do sexo feminino e dois $(4,2 \%)$ do sexo masculino; dos pacientes portadores de alguma doença neurológica, sete $(10,1 \%)$ eram do sexo feminino e três $(6,4 \%)$ do sexo masculino. A literatura especializada afirma que pacientes portadores de doenças crônicas, submetidos a procedimento cirúrgico-anestésico, apresentam maior risco de desenvolver hipotermia ${ }^{9}$.

A visita pré-operatória é realizada 24 horas antes do procedimento cirúrgico, por um Enfermeiro do Centro Cirúrgico, utilizando um instrumento de Sistematização da Assistência de Enfermagem Perioperatótia (SAEP) no leito da enfermaria e complementada por consulta ao prontuário e esclarecimentos de dúvidas existentes com a equipe multidisciplinar da unidade de internação.

O processo de recepção do paciente acontece com o acolhimento de um Enfermeiro e um Auxiliar de Enfermagem na sala de admissão. O Enfermeiro coleta os dados iniciais que auxiliarão no período intraoperatório, verifica exames e realiza avaliação de preparo específico de cada procedimento cirúrgico. O paciente é paramentado com uma touca descartável, propés e lençol, conforme rotina do serviço.

$\mathrm{Na}$ Tabela 1, são apresentados os dados sobre a temperatura na sala de admissão, a temperatura do forno, o armazenamento de fluidos e a temperatura na sala de cirurgia.

Em relação à temperatura na sala de admissão, em Celsius, a média encontrada antes de o paciente adentrar na sala de cirurgia foi de $23,3^{\circ} \mathrm{C}$ (Desvio Padrão $=0,5$ ), com variação de $22^{\circ} \mathrm{C}$ (mínima) e $25^{\circ} \mathrm{C}$ (máxima). $\mathrm{O}$ fato da temperatura na sala de admissão estar dentro dos valores recomendados pela Association of Operative Registered Nurses $(\mathrm{AORN})^{10}$ é um fator externo muito importante para promoção da normotermia. A AORN recomenda, como uma das intervenções pré-operatórias, manter a temperatura ambiente $\geq 24^{\circ} \mathrm{C}$.

A hipotermia perioperatória tem sido prevalente em pacientes idosos, em virtude de terem seu sistema termorregulador comprometido. A idade é um fator de risco para o desenvolvimento de hipotermia, sendo a idade superior a 60 anos fator preditivo para a hipotermia durante a cirurgia.. Esses pacientes, necessitam de maiores cuidados no pós-operatório ${ }^{11}$, devido a uma resposta termorreguladora diminuída, quando comparados a pacientes de a outras faixas etárias, em decorrência da alteração na resposta vasoconstrictora concebida com a idade ${ }^{12}$.

A American Society of Peri Anesthesia Nurses (ASPAN) ${ }^{13}$ orienta, para promoção da normotermia perioperatória, que se atente para a idade e o sexo feminino como fatores de risco para a hipotermia. $\mathrm{Na}$ admissão do paciente, a enfermagem deve desenvolver um plano de cuidados para minimizar o risco de hipotermia nesses pacientes. A(o) Enfermeira(o) precisa garantir um ambiente propício para o desenvolvimento do cuidado, envolvendo, dentre outros, o meio ambiente físico e social ${ }^{14}$.

A temperatura média do forno de banho-maria, em Celsius, foi de $58^{\circ} \mathrm{C}(\mathrm{DP}=5,4)$, porém foi observado que, em alguns momentos, o forno encontrava-se desligado. A temperatura mínima foi de $37^{\circ} \mathrm{C}$ e a máxima de $69^{\circ} \mathrm{C}$. A média de temperatura de armazenamento de fluidos foi de $35^{\circ} \mathrm{C}(\mathrm{DP}=6,7)$. Algumas vezes, não houve esse armazenamento, pois os profissionais usavam as soluções aquecidas imediatamente após a retirada do forno de banho-maria.

A temperatura do forno não se apresentava adequada para o aquecimento dos fluidos intravenosos e de irrigação. A AORN ${ }^{10}$ orienta que a administração de líquidos intravenosos à temperatura ambiente pode causar hipotermia e estima-se que $1 \mathrm{~L}$ de solução cristaloide à temperatura ambiente diminui a temperatura corporal $\mathrm{em} 0,25^{\circ} \mathrm{C}$. Ainda de acordo com a $\mathrm{AORN}^{10}$, a perda de calor, através da administração de fluidos endovenosos à temperatura ambiente, não é considerada como uma das formas mais significativas de redução da temperatura corporal, pois conforme estes vão sendo administrados, são aquecidos pelo sangue e pelos tecidos. Porém, é sabido que, embora a infusão dos fluidos endovenosos aquecidos não traga resultados imediatos, seu efeito é termogênico, ou seja, na sala de recuperação anestésica, há menor incidência de tremor (tempo e intensidade). Recomenda-se, ainda, que, para um resultado efetivo, esse método sempre deve estar associado a outras técnicas de aquecimento.

$\mathrm{O}$ aquecimento de fluidos diminui a perda de calor condutiva e observa-se que pacientes, ao receberem fluidos

Tabela 1. Distribuição da estatística descritiva segundo a temperatura na sala de admissão, do forno, do recipiente para armazenamento e na sala de cirurgia. Fortaleza-Ce, 2011.

\begin{tabular}{lccccc}
\hline \multicolumn{1}{c}{ Procedimento } & N & Mínimo & Máximo & Média & Desvio padrão (DP) \\
\hline Temperatura da sala de admissão & 115 & 22,0 & 25,6 & 23,36 & 0,5766 \\
Temperatura do forno & 107 & 37,00 & 69,00 & 58,1506 & 5,466 \\
Temperatura de armazenamento de fluidos & 74 & 22,6 & 42,8 & 35,018 & 6,7378 \\
Temperatura na sala de cirurgia & 120 & 18,1 & 24,3 & 20,772 & 1,1711 \\
\hline
\end{tabular}

Fonte: da pesquisa, Fortaleza-CE, 2011. 
aquecidos, têm alterações hemodinâmicas, tremores e tempo de recuperação diminuídos ${ }^{15}$.

Durante a coleta, foi observada a falta de maiores cuidados com o uso dos fluidos aquecidos. A enfermagem precisa estar mais presente neste cuidado, pois se tem percebido outros profissionais intervindo nesse cuidado e negligenciando a prevenção da hipotermia; a enfermagem deve ter, também, conhecimento de sua autonomia em administrar os fluidos aquecidos no paciente. $\mathrm{O}$ cuidado da enfermagem não está somente em aquecer os fluidos, mas também em oferecer ao paciente os fluidos aquecidos para serem administrados com segurança.

A AORN ${ }^{10}$ recomenda o aquecimento de fluidos por via intravenosa, se forem administrados em grandes volumes, ou seja, consideram-se $2 \mathrm{~L} /$ hora para adultos e devem ser aquecidos a uma temperatura de $37^{\circ} \mathrm{C}$. Observe-se, ainda, que os fluidos aquecidos devem ser usados como complemento dos métodos de aquecimento ativo, pois sendo usados sozinhos não previnem a hipotermia. A AORN ${ }^{10}$ apresenta um estudo com pacientes submetidos à laparoscopia em que foram utilizados fluidos aquecidos em altas temperaturas, sem o método de aquecimento ativo, para prevenir diminuição da temperatura dos pacientes, o que resultou em lesões nos pacientes.

No presente estudo, os fluidos eram armazenados em um recipiente de isopor após serem aquecidos em banho-maria no forno. Ao colocar o termômetro no início da tardeem seu interior, verificou-se uma temperatura de $40^{\circ} \mathrm{C}$, mas ao final da tarde essa temperatura caía para $35^{\circ} \mathrm{C}$. Ou seja, o uso de aquecimento de fluidos na instituição merece estudos e avaliações, para tornar-se um procedimento seguro de aquecimento de fluidos.

No entanto, é importante ressaltar que a técnica de banhomaria apresenta riscos de contaminação, molha o material aquecido, além de ser demorada, pois o aquecimento ocorre por contato úmido ${ }^{16}$.

De acordo com a recomendação da $\operatorname{ASPAN}^{13}$, a temperatura da sala de cirurgia deve permanecer entre $20^{\circ} \mathrm{Ce}$ $24^{\circ} \mathrm{C}$. A média da temperatura da sala de cirurgia apresentou variações discretas mantendo-se entre $18,1^{\circ} \mathrm{C}$ e $24,3^{\circ} \mathrm{C}$.
A média da temperatura de sala cirúrgica pode ter sofrido influência em virtude de algumas alterações feitas no sistema de refrigeração do hospital, observadas no decorrer da coleta, do número de pessoal em sala, do uso de equipamentos elétricos e da abertura de portas, que podem ter afetado o resultado deste estudo.

Monitorizar a temperatura corporal dos pacientes é uma intervenção muito negligenciada, talvez por falta de conhecimento dos profissionais a despeito dos efeitos deletérios causados pela hipotermia ou até mesmo por não saberem de sua autonomia para intervir na medida da temperatura do paciente. Verificar a temperatura do paciente na admissão é uma medida recomendada pela $\mathrm{ASPAN}^{13}$.

O paciente, quando exposto ao ambiente cirúrgico, perde calor pela pele para o ambiente por meio de radiação, convecção e evaporação. Esses mecanismos acarretam 90\% da perda metabólica de calor pela superfície cutânea. Em decorrência da temperatura ambiental baixa, a equipe deve estar atenta aos sinais físicos de hipotermia e desconforto térmico do paciente. Dessa forma, é fundamental a prevenção com o uso dos métodos de aquecimento mais eficazes, com a finalidade de diminuir os efeitos deletérios e o desconforto no pós-operatório. Analisando a temperatura corporal do paciente e a temperatura da sala de cirurgia, vê-se uma correlação estatisticamente significante, isto é, quanto maior a temperatura da sala, maior a temperatura corporal do paciente ${ }^{8}$.

A AORN ${ }^{11}$ indica que a temperatura da sala cirurgia determina quanto será a perda de calor do paciente pela pele por radiação, convecção e evaporação, devido ao uso de soluções antissépticas no preparo da pele.

A Tabela 2 apresenta as intervenções de enfermagem que foram realizadas em pacientes com co-morbidades e os fatores externos que podem levar o paciente a desenvolver hipotermia.

De todos os procedimentos cirúrgicos, $98 \%$ utilizaram para degermação da pele antissépticos frios. Ressalta-se que, com o uso de polivinilpirrolidona-iodo (PVPI), o paciente perde calor da superfície corporal, via evaporação,

Tabela 2. Distribuição dos pacientes com co-morbidades associadas segundo os fatores de risco externo. Fortaleza-CE, 2011.

\begin{tabular}{|c|c|c|c|c|c|c|c|c|c|c|}
\hline \multirow{2}{*}{ FATORES DE RISCO } & \multicolumn{2}{|c|}{ Cardiopatia } & \multicolumn{2}{|c|}{ Neurologia } & \multicolumn{2}{|c|}{ Hipertensão } & \multicolumn{2}{|c|}{ Diabetes } & \multicolumn{2}{|c|}{ Total } \\
\hline & Sim & Não & Sim & Não & Sim & Não & Sim & Não & $\mathbf{N}$ & $\%$ \\
\hline $\begin{array}{l}\text { Uso de líquidos frios para degermação } \\
\text { da pele }\end{array}$ & 4 & 112 & 10 & 102 & 29 & 45 & 12 & 103 & 116 & 98,3 \\
\hline $\begin{array}{l}\text { Uso de fluidos intravenosos e de irrigação } \\
\text { cavitária frios }\end{array}$ & 0 & 56 & 4 & 50 & 10 & 45 & 3 & 53 & 56 & 94,6 \\
\hline $\begin{array}{l}\text { O procedimento cirúrgico era com } \\
\text { cavidades abertas e exposição de vísceras }\end{array}$ & 2 & 48 & 4 & 42 & 14 & 36 & 5 & 45 & 50 & 47,4 \\
\hline Ambiente frio da sala cirúrgica & 4 & 108 & 9 & 100 & 27 & 84 & 11 & 99 & 112 & 32,37 \\
\hline Exposição da superfície corporal & 0 & 40 & 1 & 36 & 7 & 33 & 5 & 35 & 40 & 33,3 \\
\hline
\end{tabular}

Fonte: da pesquisa, Fortaleza-CE, 2011. 
especialmente quando os antissépticos tiverem conteúdo alcóolico9.

Os profissionais de enfermagem do perioperatório devem compreender a responsabilidade do cuidado ao aquecer os antissépticos que serão utilizados para fazer degermação na pele do paciente. Os profissionais devem incentivar a instituição hospitalar a investir em dispositivos eficientes, econômicos, práticos e rápidos no aquecimento dos degermantes. $\mathrm{O}$ uso destes produtos em temperatura ambiente favorece a perda de calor da superfície corporal.

Segundo a AORN ${ }^{10}$, alguns distúrbios metabólicos inibem a termorregulação, impedindo a produção de calor ou respostas fisiológicas às mudanças de temperatura externa. Assim, disfunções do sistema nervoso e cardiológico também podem comprometer a produção de calor no paciente. A enfermagem deve desenvolver um plano de cuidados aos pacientes considerados de risco para minimizar a probabilidade da ocorrência de hipotermia perioperatória. Os métodos de aquecimento mais eficazes devem ser empregados para prevenir a hipotermia; verificar a temperatura e avaliar o conforto térmico desses pacientes constituem medidas seguramente preventivas para o desenvolvimento de hipotermia.

Em procedimento cirúrgico com cavidades abertas e exposição de vísceras de pacientes hipertensos, foi encontrado um quantitativo significativo de eventos que necessitavam de ações de prevenção da hipotermia. Nesses procedimentos, a AORN ${ }^{10}$ recomenda utilizar fluidos de irrigação aquecidos a uma temperatura de $37^{\circ} \mathrm{C}$ e verificar a temperatura do soro com um termômetro no momento da utilização do mesmo.

Para a variável 'ambiente frio', considera-se frio quando a temperatura da sala cirúrgica for menor do que é determinado pela $\mathrm{AORN}^{10}$ (entre $20^{\circ} \mathrm{C}$ e $24^{\circ} \mathrm{C}$ ) e quando o paciente apresentou tremores ou verbalizou desconforto térmico.

A AORN ${ }^{10}$ recomenda que a temperatura em sala deve ser aumentada quando o aquecimento ativo não é viável ou quando aquecimento ativo não é suficiente para aquecer o paciente, devendo a sala estar com uma temperatura ambiente acima de $23^{\circ} \mathrm{C}$; a equipe de enfermagem deve estar ciente de que esse fator de risco externo associado a outros fatores implicará em complicações pós-operatórias e retardo de alta hospitalar.

A Tabela 3 mostra que, na observação feita no transoperatório pela pesquisadora, há ausência do Enfermeiro na intervenção acerca do conforto térmico do paciente. A intervenção sobre o conforto térmico tem como fim a manutenção e a preservação da vida e do bem-estar, contribuindo para uma assistência que atenda às necessidades de conforto e diminuição da ansiedade do paciente ${ }^{16}$. A dimensão dessa intervenção deve ser valorizada e é necessária para a prática de enfermagem no perioperatório.

A instituição hospitalar tem condições tecnológicas para os profissionais de enfermagem intervir nessa prática, pois cada sala cirúrgica possui monitor com termômetro de nasofaringe. Pode-se supor, desta forma, que a ausência desta intervenção se relaciona a falta de conhecimento da importância emse verificar um sinal vital que, se não for avaliado com cuidado, pode acarretar consequências deletérias da hipotermia.

AAORN ${ }^{10}$ recomenda verificar a temperatura central com o termômetro de nasofaringe, pois o dispositivo fornece uma leitura mais precisa, confiável e não sensível à temperatura exterior. Para isso, é importante o profissional assegurar-se da confiabilidade do dispositivo de acessibilidade, para segurança do paciente e facilidade de uso.

A Tabela 4 demonstra que o método de aquecimento utilizado por 70,4\% dos Enfermeiros e 81,5\% dos Técnicos foi o cobertor de lã associado ao lençol de algodão, ao sair da sala de cirurgia. $O$ emprego da manta térmica neste estudo foi reduzido: apenas quatro Enfermeiros e 11 Técnicos usaram esse dispositivo nos 120 procedimentos cirúrgicos investigados.

Estudos anteriores demonstraram que os pacientes aquecidos com o sistema de ar forçado demoraram, em média, 195 minutos para se reaquecerem; os que utilizaram o colchão de água aquecido, 220 minutos, e os que fizeram uso de manta de algodão demoraram, em média, 295 minutos. Assim, o sistema de ar forçado aquecido foi o método de aquecimento mais efetivo, justificando seu emprego preferivelmente em pacientes hipotérmicos, em detrimento de métodos de aquecimento passivo ${ }^{18}$.

A implementação de métodos para a manutenção da normotermia do paciente perioperatório é crucial. Neste cenário, compete ao Enfermeiro a implantação de intervenções eficazes que proporcionam a prevenção ou o tratamento da hipotermia, e a diminuição das complicações associadas a este evento.

Em 2007, a Association of PeriOperative Registered Nurses $^{13}$ atualizou e publicou as recomendações práticas para a prevenção e o tratamento da hipotermia no perioperatório,

Tabela 3. Distribuição dos profissionais que realizaram a intervenção de questionar o paciente acerca de seu conforto térmico. Fortaleza-CE, 2011.

\begin{tabular}{|c|c|c|c|c|c|c|c|}
\hline \multirow{3}{*}{$\begin{array}{c}\text { Procedimento } \\
\text { Questionou o paciente acerca } \\
\text { de seu conforto térmico }\end{array}$} & \multicolumn{4}{|c|}{ Categoria } & \multicolumn{3}{|c|}{ Total } \\
\hline & \multicolumn{2}{|c|}{ Enfermeiro } & \multicolumn{2}{|c|}{ Técnico } & \multirow{2}{*}{$\mathbf{N}$} & \multirow{2}{*}{$\%$} & \multirow{2}{*}{$\mathbf{p}$} \\
\hline & $\mathbf{N}$ & $\%$ & $\mathbf{N}$ & $\%$ & & & \\
\hline Sim & 1 & 4,2 & 9 & 10,5 & 10 & 9,1 & \\
\hline Não & 23 & 95,8 & 77 & 89,5 & 100 & 90,9 & \\
\hline Total & 24 & 100 & 86 & 100 & 110 & 100 & 0,304 \\
\hline
\end{tabular}


Tabela 4. Distribuição dos profissionais quanto ao uso do método utilizado para aquecer o paciente. Fortaleza, março e junho/2011.

\begin{tabular}{|c|c|c|c|c|c|c|c|}
\hline \multirow{3}{*}{$\begin{array}{l}\text { Verificou temperatura central } \\
\text { durante todo o procedimento cirúrgico }\end{array}$} & \multicolumn{4}{|c|}{ Categoria } & \multirow{2}{*}{\multicolumn{3}{|c|}{ Total }} \\
\hline & \multicolumn{2}{|c|}{ Enfermeiro } & \multicolumn{2}{|c|}{ Técnico } & & & \\
\hline & $\mathbf{N}$ & $\%$ & $\mathbf{N}$ & $\%$ & $\mathbf{N}$ & $\%$ & $\mathbf{P}$ \\
\hline Sim & 2 & 7,7 & 2 & 2,4 & 4 & 3,7 & \\
\hline Não & 24 & 92,3 & 81 & 97,6 & 105 & 96,3 & \\
\hline Total & 26 & 100 & 83 & 100 & 109 & 100 & 0,248 \\
\hline
\end{tabular}

Fonte: da pesquisa, Fortaleza-CE, 2011.

apontando a necessidade de avaliação da temperatura corporal do paciente em todas as fases deste período, ou seja, no pré, intra e pós-operatório, bem como a importância da implementação de métodos passivo e ativo de aquecimento.

$\mathrm{O}$ avanço da tecnologia apresenta os novos métodos de aquecimento cutâneo, composto de dois sistemas com uma unidade geradora de água aquecida, que circula por mangueiras para dispositivos de uso único, sendo que, no sistema de circulação de água aquecida, os dispositivos podem ser usados em diferentes segmentos do corpo do paciente, como tronco e/ou membros superiores e inferiores. Os dispositivos do outro sistema são adesivos, hidrofílicos de gel e flexíveis, e podem cobrir abdome, dorso e coxas do paciente, acarretando uma interface íntima com a pele, o que permite uma ótima transferência de energia da unidade geradora de água para o paciente . $^{8}$.

A falta de diretrizes baseadas em evidências torna as decisões sobre como aquecer o paciente baseadas em tradição e conveniência, que são difíceis de serem mudadas ${ }^{20}$.

\section{Conclusão}

A presente pesquisa permitiu concluir que a temperatura na sala de admissão foi menor que a recomenda pela AORN e a temperatura do forno, o armazenamento de fluidos e a temperatura na sala de cirurgia não estão dentro dos valores recomendados pela mesma.

Os antissépticos utilizados nos pacientes com comorbidades não são aquecidos, bem como os fluidos intravenosos e de irrigação cavitária.

Os procedimentos cirúrgicos com pacientes portadores de doenças crônicas foram realizados quando a sala cirúrgica se encontrava fria. Apenas um Enfermeiro e nove Técnicos questionaram o paciente acerca de seu conforto térmico. Nenhum profissional de enfermagem verificou a temperatura central durante todo procedimento cirúrgico. Enfatiza-se a intervenção de dois profissionais - sem ser da enfermagem - para verificar a temperatura central do paciente.

Em relação às limitações do estudo, compreendemos que, devido ao tamanho da amostra, a generalização dos resultados deve ser feita com mais prudência.

Alguns procedimentos, como questionar o paciente acerca de seu conforto térmico, não foram observados, em virtude de ter de estar em mais de uma sala ao mesmo tempo, o que trouxe limitaçõesao estudo.

Buscando-se a preservação e a segurança do paciente no transoperatório, a profilaxia da hipotermia acidental pela enfermagem exerce papel fundamental, pois se sabe que aproximadamente $90 \%$ da perda de calor do organismo, durante o ato cirúrgico, ocorre pela superfície da pele, através de irradiação e convecção, associadas à evaporação no sítio cirúrgico.

Para que seja efetivo, qualquer dispositivo, que previna ou trate a hipotermia, deverá reduzir a perda de calor do organismo. O caminho inicial para se reduzir a perda de calor pela superfície corpórea está no controle da temperatura ambiente, no uso de dispositivos ativos e passivos de manutenção da temperatura do paciente, no controle e na avaliação clínica do paciente, e na manutenção de temperatura dos fluidos intravenosos.

Deve-se acompanhar a evolução dos estudos publicados baseando-se a prática clínica em evidências científicas, para a prevenção da hipotermia acidental no transoperatório.

\section{Referências}

1. Santos ST, Caregnato RCA. Hipotermia acidental perioperatória: proposta de protocolo de assistência de enfermagem. Rev SOBECC. 2010;15(2):45-5.

2. Zappelini CEM, Sakae CEM, Mamôru T, Bianchini N, Brum SPB. Avaliação de hipotermia na sala de recuperação pós-anestésica em pacientes submetidos a cirurgias abdominais com duração maior de duas horas. ACM Arq Catarin Med. 2008;37(2):25-31.

3. Bernardis RCG, Silva MP, Gozzani JL, Pagnocca ML, Mathias LAST. Uso da manta térmica na prevenção da hipotermia intraoperatória. Rev Assoc Med Bras. 2009;55(4):421-6. PMid:19750309. http:// dx.doi.org/10.1590/S0104-42302009000400017

4. Pagnocca ML, Tai EJ, Dwan JL. Controle de temperatura em intervenção cirúrgica abdominal convencional: comparação entre os métodos de aquecimento por condução e condução associada à convecção. Rev. Bras. Anestesiol 2009;59(1):56-66. PMid:19374216.

5. Golin V, Sprovieri SRS, Bedrikow R, Pereira AC, Melhado VER, Salles MJC, et al. Hipotermia acidental em um país tropical. Rev Assoc Med Bras. 2003;49(3):261-5. PMid:14666349. http://dx.doi. org/10.1590/S0104-42302003000300028

6. Biazzotto CB, Brudniewski M, Schmidt AP, Auler JOC Jr. Hipotermia no período peri-operatório. Rev Bras Anestesiol. 
2006;56(1):89-106. PMid:19468555. http://dx.doi.org/10.1590/ S0034-70942006000100012

7. Poveda VB, Piccoli M, Galvão CM, Sawada NO. Métodos de prevenção e reaquecimento do paciente para o perioperatório. Rev Eletr Enf. 2005;7(3):266-72.

8. Poveda VB, Galvao CM, Santos CB. Fatores relacionados ao desenvolvimento de hipotermia no período intra-operatório. Rev Latino-Am Enferm. 2009;17(2):228-33.

9. Tramontini CC, Graziano KU. Controle da hipotermia de pacientes cirúrgicos idosos no intraoperatório: avaliação de duas intervenções de enfermagem. Rev Latino-Am Enferm. 2009;15(4):626-31. http://dx.doi.org/10.1590/S0104-11692007000400016

10. Association of Operating Room Nurses - AORN. Recommend practices for the prevention of unplanned perioperative hypothermia. AORN J. 2007;85(5):972-88.

11. Bitner, Hilde L, Hall K, Duvendack T. A Team Approach to the Prevention of Unplanned Postoperative Hypothermia. AORN J. 2007;85(5):921-9. PMid:17499055. http://dx.doi.org/10.1016/j. aorn.2007.04.009

12. Kasai, Hirose M, Yaegashi K, Matsukawa T, Takamata A, Tanaka Y. Preoperative Risk Factors of Intraoperative Hypothermia in Major
Surgery Under General Anesthesia. Anesth Analg. 2002;95(5):13813. http://dx.doi.org/10.1097/00000539-200211000-00051

13. American Society of Perinesthesia Nurses - ASPAN. Clinical guiadeline for the prevention of unplanned perioperative hypothermia. J Perianesth Nurs. 2001;16(5):305-14. http://dx.doi. org/10.1053/jpan.2001.28060

14. Silva DC, Alvim NAT. Ambiente do centro cirúrgico e os elementos que o integram: implicações para os cuidados de enfermagem. Rev Bras Enf. 2010;63(3):427-34. http://dx.doi.org/10.1590/ S0034-71672010000300013

15. Hasankhani $\mathrm{H}$. The effects of intravenous fluids temperature on perioperative hemodynamic situation, post-operative shivering, and recovery in orthopaedic surgery. Can Oper Room Nurs J. 2005;6(2):7-11.

16. Pisani IS. Prevenção da hipotermia Per-Operatória e a utilidade do forno de microondas. Rev Bras Anestesiol. 1999;49(6):399-402.

17. Gotardo JM, Galvão, CM. Avaliação da Hipotermia no pósoperatório imediato. Rev REME. 2009;10(2):113-21.

18. Weirich TL. Hypothermia/Warming Protocols: Why Are They Not Widely Used in the OR?. AORN J. 2008;87(2):333-4. PMid:18262000. http://dx.doi.org/10.1016/j.aorn.2007.08.021 